\title{
BIFOCAL NEURAL ASR: EXPLOITING KEYWORD SPOTTING FOR INFERENCE OPTIMIZATION
}

\author{
Jonathan Macoskey, Grant P. Strimel, Ariya Rastrow
}

\author{
Amazon.com \\ \{macoskey, gsstrime, arastrow $\}$ eamazon.com
}

\begin{abstract}
We present Bifocal RNN-T, a new variant of the Recurrent Neural Network Transducer (RNN-T) architecture designed for improved inference time latency on speech recognition tasks. The architecture enables a dynamic pivot for its runtime compute pathway, namely taking advantage of keyword spotting to select which component of the network to execute for a given audio frame. To accomplish this, we leverage a recurrent cell we call the Bifocal LSTM (BFLSTM), which we detail in the paper. The architecture is compatible with other optimization strategies such as quantization, sparsification, and applying time-reduction layers, making it especially applicable for deployed, real-time speech recognition settings. We present the architecture and report comparative experimental results on voice-assistant speech recognition tasks. Specifically, we show our proposed Bifocal RNN-T can improve inference cost by $29.1 \%$ with matching word error rates and only a minor increase in memory size.
\end{abstract}

Index Terms - On-device speech recognition, recurrent neural network transducer (RNN-T), inference optimization.

\section{INTRODUCTION}

The increasing omnipresence of smartphones, smart speakers, and tablets coupled with the adoption of voice assistants has motivated a modern trend to develop Automatic Speech Recognition (ASR) systems which fully operate on local devices [1, 2] 3]. The promise of on-device ASR includes increased reliability, improved latency and privacy benefits by alleviating the need to stream audio to servers. In order to realize these benefits, however, new approaches are required to address the challenges posed by compute constrained devices.

A complementary trend prevalent in the literature is a shift from traditional Hidden Markov Model (HMM)-based ASR systems to end-to-end approaches [4, 5, 6, 7, 8]. These end-to-end architectures replace the typically disjoint components in an ASR system with a single, fully neural architecture trained over large amounts of data.

The fully neural approaches are strong candidates for lowfootprint settings due to their simplicity and uniform compression ability; however, when deployed on devices with hardware constraints, e.g. limited compute and memory bandwidth, they still require careful use of compression algorithms and optimization techniques to achieve real-time, low-latency speech recognition.

In order to address this latency bottleneck, several engineering and modeling techniques have been proposed, especially for streaming architectures such as RNN-T [4]. Several studies [9, 10, 11] have investigated moving from 32-bit weights down to 8 or 4 bits for neural ASR models. These quantized networks not only reduce model size but also eliminate costly floating point operations and can better integrate with hardware [12]. Likewise, experiments have shown that low-rank matrix factorization [13] and network sparsification methods [13, 14] can be applied to an extent without significant accuracy degradation. Reducing parameter operations and complexity leads to inference cost gains for these methods.

Higher-level architectural changes designed to improve inference speed have also been proposed [15]. The RNN topologies of the CIFG-LSTM [16], which has 25\% fewer parameters compared to the standard LSTM [17], and Simple Recurrent Units (SRU) [18] are suggested as alternative recurrent layers for on-device ASR. These authors show a retention in modeling capacity with fewer parameters and less compute required at runtime. Finally, there are common latency reduction methods, such as time-reduction [7, 8, 19], which reduce the effective decoding frame rate at which audio is processed.

In this work, we present a new variant of the RNN-T architecture, Bifocal RNN-T, which is designed to lower latency for on-device ASR applications. The architecture leverages design characteristics common to voice assistant applications, specifically keyword spotting. Voice assistants will typically use a lightweight, continuously-running keyword spotter model strictly to detect a pre-determined wake word (WW) or phrase (e.g. "Alexa" or "Ok Google") [20, 21]. For efficiency and privacy, ASR decoding is deferred until WW recognition, at which point ASR begins decoding streamed audio along with a buffered lead-in segment of pre-WW audio. While this design provides a natural user experience with additional robustness built in (i.e. lowering the false-accept rate by re-verifying the presence of the WW during ASR), the buffering introduces a latency hurdle which generic techniques do not address. Our Bifocal RNN-T architecture is constructed to address this problem by incorporating keyword spotting as part of its design. Our contributions are complementary to the existing literature and can be used in conjunction with the group of optimizations referenced. The remainder of the paper is organized as follows. Sections 2 and 3 detail the Bifocal RNN-T and its critical components, namely the Bifocal LSTM. Section 4 extends the basic approach further while Section 5 outlines experiments that show the benefits of Bifocal RNN-T on speech recognition tasks.

\section{BIFOCAL RNN-T}

The Recurrent Neural Network Transducer (RNN-T) [4] is a fully neural, sequence-to-sequence architecture that is particularly applicable for ASR modeling. The architecture is trained end-to-end with 
what can be viewed as three logical components: the encoder (also referred to as the transcription network), the decoder (also referred to as the prediction network), and an optional joint network. For ASR, the transcription network $\mathcal{F}$ is a multi-layer RNN, which operates over feature vectors $x_{1: T}$ extracted from a raw audio signal. For speech, these will be features given by transformations over the signal (e.g. log-mel filter bank) and stacking consecutive samples into frames. The encoder outputs a vector $h_{t}^{\text {enc }}$ for each frame of the input based on the sequence of frames observed up to that point:

$$
h_{t}^{\text {enc }}=\mathcal{F}\left(x_{1: t}\right) \text {. }
$$

The decoder $\mathcal{G}$ is also a multi-layer $\mathrm{RNN}$ which operates over the sequence of outputted labels $y_{1: M}$. The labels in our setting are word pieces. The decoder computes a new vector $h_{m}^{\mathrm{dec}}$ for each new outputted label of the RNN-T:

$$
h_{m}^{\mathrm{dec}}=\mathcal{G}\left(y_{1: m}\right) \text {. }
$$

The prediction network embodies the role of the language model in a traditional HMM hybrid system, operating over labels to compute scores to be combined with the output from $\mathcal{F}$. This operation is the role of the joint network $\mathcal{J}$, which serves either as (i) a simple additive operation between the outputted vectors of each network [4] or (ii) as a feedforward network [2], to construct a probability distribution for the next output label:

$$
\mathcal{J}\left(h_{t}^{\mathrm{enc}}, h_{m}^{\mathrm{dec}}\right)=\left\{\begin{array}{l}
h_{t}^{\mathrm{enc}}+u_{m}^{\mathrm{dec}} \\
\psi\left(W h_{t}^{\mathrm{enc}}+V h_{m}^{\mathrm{dec}}\right)
\end{array}\right.
$$

where $\psi$ is an activation. Finally, the conditional output distribution $P\left(\hat{y}_{m+1} \mid x_{1: t}, y_{1: m}\right)$ is obtained by applying a softmax to the result of $\mathcal{J}$. For real-time speech settings, the $\mathcal{F}$ and $\mathcal{G}$ are commonly built by stacking multiple unidirectional LSTMs [2] and the network is trained with what amounts to an extension of the Connectionist Temporal Classification (CTC) approach [22], allowing RNN-T to implicitly learn to align audio and transcriptions.

Voice assistant enabled devices, like Amazon Echo and Google Home, use keyword spotting as a central design element of their user interface and entry point to ASR model decoding. A small, highly accurate keyword spotting model is used to continuously process audio on-device and, when appropriate, activate streaming audio through the ASR module. A voice assistant system, however, can buffer a segment of audio to allow any "lead-in" words spoken before the WW (and including WW itself) to also be decoded by the ASR module. For example, in utterances "Hey Alexa, turn on the light." or "Hello Echo, is it raining?", the lead-in segment would be "Hey Alexa" or "Hello Echo", respectively.

Here we propose a modified RNN-T architecture which takes advantage of the keyword signal for low compute settings. We call our approach Bifocal RNN-T because the architecture has multiple "lenses" designed to focus on different segments of an utterance. The distinguishing feature of the design is training two alternative encoder RNNs and leveraging the keyword spotting to pivot between the two. A small $\mathcal{F}^{s}$ encoder network is trained for the lead-in segment and a large $\mathcal{F}^{\ell}$ encoder network is trained for processing the remainder of the audio. While sharing an equal number of layers, $\mathcal{F}^{s}$ has a smaller number of hidden units than $\mathcal{F}^{\ell}$, allowing a faster but coarser processing of frames. $\mathcal{F}^{\ell}$, in contrast, will have a higher capacity but at the cost of a higher compute. The natural lower perplexity of the spoken words prior to the WW lends itself to using

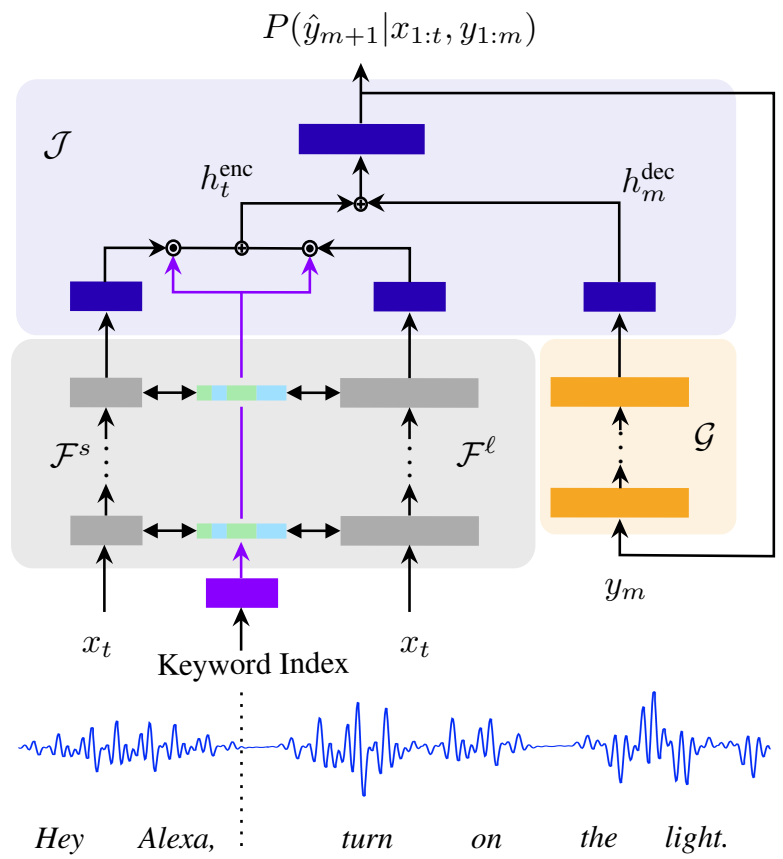

Fig. 1: Bifocal RNN-T architecture. Two encoder networks (a small $\mathcal{F}^{s}$ and large $\mathcal{F}^{\ell}$ ) are trained using BF-LSTM cells (gray), see Section 3 Their mutually exclusive execution is determined by the frame index signaling the end of the WW (purple). The hidden state is transitioned between encoders after the WW. The decoder (gold) and the joint network (blue) operate as in standard RNN-T.

a lighter-weight model while the higher perplexity of the post-WW utterance lends itself to using a model with greater capacity. The result of employing this dual-encoder Bifocal architecture is that we obtain a larger model with a lowered total compute cost summed over all frames of an utterance. Note that the Bifocal design places an emphasis on reducing the encoder inference cost since it is a primary bottleneck during runtime; the encoder is larger, executed every frame, and not cacheable unlike the decoder which is smaller, requires fewer queries (which also can be parallelized across different threads), and whose results are cacheable during decoding.

Because the lead-in segment is buffered, it becomes critical to process this audio backlog rapidly in order for the processing to catch-up and finish decoding in real-time. Failure to do so risks perpetual lag during inference, which results in a significant response latency. Moreover, the lead-in segment will typically amount up to $30 \%$ of the total audio.

Figure 1 depicts the Bifocal RNN-T with its two encoder branches whose execution is toggled by the frame index denoting the completion of the WW, where the index is passed to the ASR module from the upstream keyword spotter component. We build the $\mathcal{F}^{s}$ and $\mathcal{F}^{\ell}$ encoders with a specialized LSTM cell, detailed in the next section, which enables us to train both networks in unison and provide a mechanism to smoothly transition the hidden state of the RNN-T network during the switch after the WW.

\section{A SWITCHING LSTM CELL}

The critical component of the Bifocal RNN-T is the Bifocal LSTM (BF-LSTM). The BF-LSTM is a trainable RNN cell that enables dynamic inference pathways at runtime. The design is implemented by 


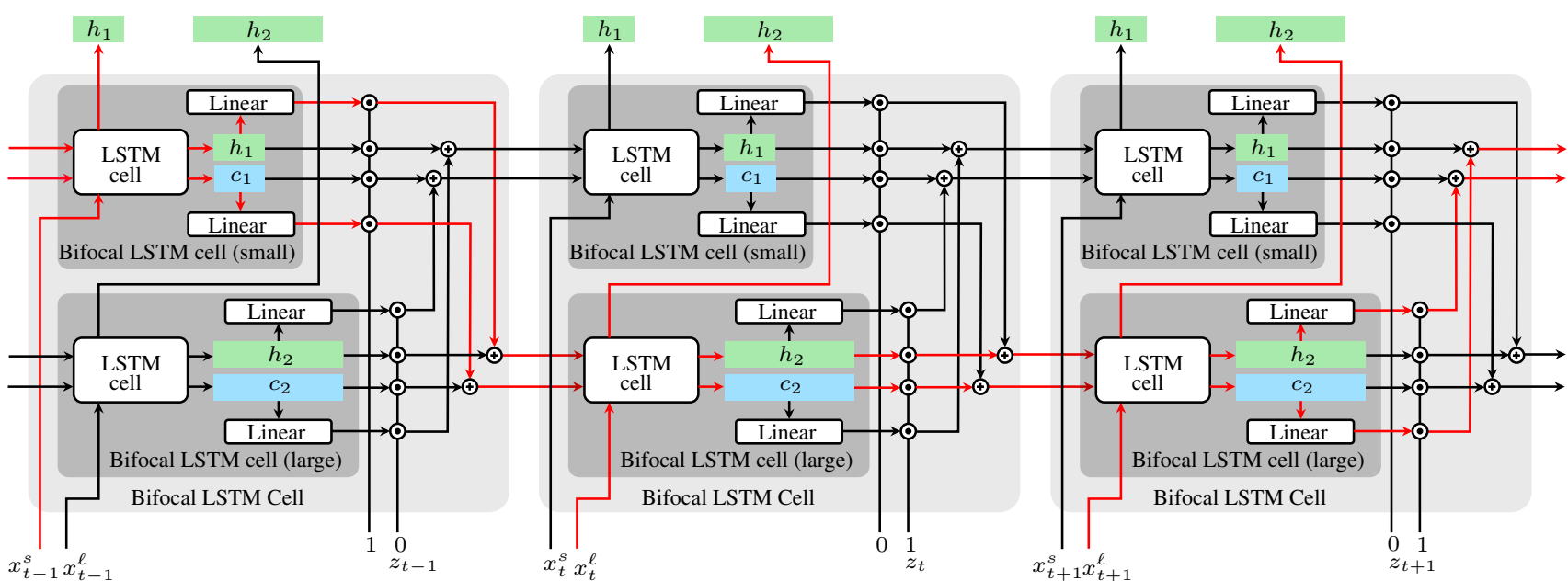

Fig. 2: Unfolded Bifocal LSTM through time. Each block represents the execution of the Bifocal LSTM Cell at single time step. While the layer is fully connected and differentiable, only one component LSTM cell is executed at each timestep during inference. This is highlighted by the red pathways and is controlled by the switching signal $z_{t}$.

training two distinct LSTM cells per layer that learn to transfer their hidden states into one another by projection. We refer to these two cells working in tandem within the BF-LSTM as the small and large cells. Their names reference their relative dimensionality. The small cell has fewer units, which amounts to a faster inference with a less granular modeling function. The large cell possesses a greater capacity but at the cost of more parameters on which to operate. These cells have different hidden state sizes, so to switch between them at different frames, we add learned "translation" operations for projecting into each others' state spaces. Switching at each timestep is controlled by a user-provided input $z_{t} \in\{0,1\}$ to signal the small $\left(z_{t}=0\right)$ and large $\left(z_{t}=1\right)$ cells. We present the equations for the BF-LSTM below (note the affine transforms are given without their bias for brevity but can be included).

$$
\begin{aligned}
& \overbrace{f_{t}^{s}=\sigma\left(W_{f}^{s} x_{t}^{s}+U_{f}^{s} h_{t-1}^{s}\right)}^{\text {Standard LSTM ( } s \text { mall) }} \\
& \overbrace{f_{t}^{\ell}=\sigma\left(W_{f}^{\ell} x_{t}^{\ell}+U_{f}^{\ell} h_{t-1}^{\ell}\right)}^{\text {Standard LSTM ( } \text { arge })} \\
& i_{t}^{s}=\sigma\left(W_{i}^{s} x_{t}^{s}+U_{i}^{s} h_{t-1}^{s}\right) \\
& i_{t}^{\ell}=\sigma\left(W_{i}^{\ell} x_{t}^{\ell}+U_{i}^{\ell} h_{t-1}^{\ell}\right) \\
& o_{t}^{s}=\sigma\left(W_{o}^{s} x_{t}^{s}+U_{o}^{s} h_{t-1}^{s}\right) \\
& o_{t}^{\ell}=\sigma\left(W_{o}^{\ell} x_{t}^{\ell}+U_{o}^{\ell} h_{t-1}^{\ell}\right) \\
& \tilde{c}_{t}^{s}=\tanh \left(W_{c}^{s} x_{t}^{s}+U_{c}^{s} h_{t-1}^{s}\right) \\
& c_{t}^{s}=f_{t}^{s} \circ c_{t-1}^{s}+i_{t}^{s} \circ \tilde{c}_{t}^{s} \\
& h_{t}^{s}=o_{t}^{s} \circ \sigma\left(c_{t}^{s}\right) \\
& \tilde{c}_{t}^{\ell}=\tanh \left(W_{c}^{\ell} x_{t}^{\ell}+U_{c}^{\ell} h_{t-1}^{\ell}\right) \\
& c_{t}^{\ell}=f_{t}^{\ell} \circ c_{t-1}^{\ell}+i_{t}^{\ell} \circ \tilde{c}_{t}^{\ell} \\
& h_{t}^{\ell}=o_{t}^{\ell} \circ \sigma\left(c_{t}^{\ell}\right) \\
& \begin{array}{c}
\overbrace{\hat{c}_{t}^{\ell}=P_{c}^{s} c_{t}^{s}}^{\text {State Projections }} \hat{c}_{t}^{s}=P_{c}^{\ell} c_{t}^{\ell} \\
\hat{h}_{t}^{\ell}=P_{h}^{s} h_{t}^{s} \quad \hat{h}_{t}^{s}=P_{h}^{\ell} h_{t}^{\ell}
\end{array} \\
& c_{t}^{s}:=c_{t}^{s}\left(1-z_{t}\right)+\hat{c}_{t}^{s} z_{t} \quad c_{t}^{\ell}:=\hat{c}_{t}^{\ell} z_{t}+c_{t}^{\ell}\left(1-z_{t}\right) \\
& h_{t}^{s}:=h_{t}^{s}\left(1-z_{t}\right)+\hat{h}_{t}^{s} z_{t} \quad h_{t}^{\ell}:=\hat{h}_{t}^{\ell} z_{t}+h_{t}\left(1-z_{t}\right)
\end{aligned}
$$

The learned state projection matrices unique to the BF-LSTM cell, $P_{c}^{s}, P_{h}^{s} \in \mathbb{R}^{h^{s} \times h^{\ell}}$ and $P_{c}^{\ell}, P_{h}^{\ell} \in \mathbb{R}^{h^{\ell} \times h^{s}}$, produce the translated states $\hat{c}_{t}^{\ell}, \hat{h}_{t}^{\ell} \in \mathbb{R}^{h^{\ell}}$ and $\hat{c}_{t}^{s}, \hat{h}_{t}^{s} \in \mathbb{R}^{h^{s}}$, respectively, which are toggled and then combined to rewrite the state vectors based on the switching variable $z_{t}$ designating the path of inference execution at timestep $t$.

The above construction is a fully differentiable recurrent layer which allows for straightforward training with standard backpropagation through time methods with all pathways computed. However, during inference, only one of the sub-cells' computation needs to be executed on each frame based on $z$. Furthermore, the state projection operations only need to be carried out during a switch where $z_{t} \neq z_{t+1}$. Figure 2 shows the fully connected unit through time and highlights a single pathway for inference.

One observes that there are no restrictions on the input dimensions (for $x^{s}$ and $x^{\ell}$ ) and output dimensions (for $h^{s}$ and $h^{\ell}$ ); all sizes can differ. We use this inherent flexibility to build our Bifocal RNN-T encoders by simply stacking several BF-LSTM layers in sequence. While the first layer has matching input dimensions of the audio features, the output dimensions (and thus input dimension of subsequent layers) will have non-matching dimensions. A final projection layer is used to map the two outputs from the encoder branches onto a matching dimensionality. The projected outputs are likewise combined using the $z_{t}$ switch to forward a single output to be used for the joint network.

\section{INTERLEAVING}

While we have thus far presented a design for switching based on pre/post-WW, the BF-LSTM, as seen in Section 3 is able to train over arbitrary switching patterns that translate in both directions. Moreover, the BF-LSTM design can be generalized beyond just two pathways. At the cost of additional projection parameters, the method can support an arbitrary number of branches. We therefore extended our Bifocal RNN-T approach to a regime we term interleaving.

Interleaving still relies on a small encoder network for leadin processing, but we also attempt to train a set of encoders $\mathcal{F}^{\ell_{1}}, \mathcal{F}^{\ell_{2}}, \ldots, \mathcal{F}^{\ell_{k}}$ which are interchangeable for post-WW processing. While additionally increasing the overall size of the model, the dimensionality of these encoders and their execution schedule can be chosen to lower the compute requirements across the full sequence frames. In a "Trifocal" setting, which we adopt for our experiments, a large encoder $\mathcal{F}^{\ell_{l}}$ and small encoder $\mathcal{F}^{\ell_{s}}$ share the post-WW processing burden by switching back-and-forth according to a predetermined pattern. For example, the schedule $\left(\ell_{l}, \ell_{l}, \ell_{s}, \ell_{s}\right)$ 
would cycle through each consecutive sequence of four frames by using $\mathcal{F}^{\ell_{l}}$ for two frames, then projecting to use $\mathcal{F}^{\ell_{s}}$ for two frames before projecting back to $\mathcal{F}^{\ell_{l}}$ 's state space to repeat the pattern. In Section 5. we experiment with several of these schedules and report their improved inference cost but adverse impact on the model's predictive performance. We note here that interleaving shares a similar approach to those presented in [23, 24] which leverage variable compute for RNN applications such as text-based character prediction and music modeling.

\section{EXPERIMENTAL RESULTS}

We investigate the model performance for the Bifocal RNN-T architecture on a production voice assistant ASR task. The models are trained using teacher forcing with $42 \mathrm{k}$ hours of audio consisting of de-identified utterances of far-field, English-locale, virtual assistant tasks. Tasks span all Alexa domains including contacts, home automation, music, etc. The data consists only of utterances with the WW present, labeled with a WW frame index generated by a pre-existing keyword spotting model. An average of $31.8 \%$ of each utterance consists of lead-in segment data. A typical utterance in this dataset consists of 260 frames of audio sampled at $16 \mathrm{kHz}$. Acoustic features are extracted using log-Filterbank Energies (LBFE) with 64 dimensions. Feature frames are downsampled by a factor of 3 and are stacked with a stride size of 2 to produce an overall frame size of $30 \mathrm{~ms}$.

Our baseline RNN-T model is built with five LSTM encoder layers with 1024 units per layer, two LSTM decoder layers with 1024 units per layer, and an additive joint network with no trainable parameters resulting in an $\mathrm{RNN}-\mathrm{T}$ model with $63.5 \mathrm{M}$ total parameters, $42.7 \mathrm{M}$ of which belong to the encoder. Word piece tokens were generated by extracting a vocabulary of the $4 \mathrm{k}$ most frequent subword units (plus a blank symbol) using a unigram language model [25]. We also build a smaller baseline model with an encoder using 852 units per encoder layer which will match the computational cost of our Bifocal model.

We compare against a Bifocal RNN-T, which includes the above specifications of our baseline RNN-T (using BF-LSTM in place of LSTM encoder layers) plus a second encoder network, the lead-in encoder, $\mathcal{F}^{s}$, consisting of five 256-unit BF-LSTM layers. As described in Section 2 the lead-in encoder is only used during the lead-in portion of an utterance. After the lead-in segment of an utterance, the encoder states are projected into the dimension of the larger, 1024-unit encoder, $\mathcal{F}^{\ell}$, which evaluates the remainder of an utterance. In this configuration, the projection layers are used only once during an utterance. We also include results for a Bifocal RNN$\mathrm{T}$ without the use of state projections in order to measure their impact on predictive performance against basic zero initialization. Last, we train Trifocal RNN-T models with different interleaving patterns. Each Trifocal model consists of all components of the above Bifocal RNN-T and also includes a third encoder network, $\mathcal{F}^{\ell_{s}}$, consisting of five 256-unit BF-LSTM layers. For all Trifocal models, the leadin encoder is used during the lead-in segment of an utterance, after which the states are projected into the large encoder, which evaluates the next frame. For the remainder of an utterance, frames are evaluated by either the larger $\mathcal{F}^{\ell_{l}}$ or smaller $\mathcal{F}^{\ell_{s}}$ post-WW encoders according to a pre-defined schedule. Three Trifocal schedules we tested are shown in Table 1

Each model is evaluated on a hold out test set of utterances

\begin{tabular}{ll}
\hline Model & Schedule \\
\hline Trifocal A & $\left(\ell_{l}, \ell_{l}, \ell_{s}, \ell_{s}\right)$ \\
Trifocal B & $\left(\ell_{l}, \ell_{s}, \ell_{s}\right)$ \\
Trifocal C & $\left(\ell_{l}, \ell_{l}, \ell_{s}, \ell_{s}, \ell_{s}, \ell_{s}\right)$ \\
\hline
\end{tabular}

Table 1: Trifocal model schedules.

for virtual assistant tasks. Our predictive metric is word error rate (WER), and we decode using a standard beam search with a beam size of 16. To measure compute cost, we calculate the total number of encoder floating point operations (FLOPs) required to analyze each utterance. This calculation includes the total number of operations processed by each encoder in addition to the operations associated with the projection of state variables where required. Again, our focus on the encoder is because it presents the critical latency bottleneck for real-time decoding. The performance and compute cost for each model are shown in Table 2

\begin{tabular}{lrrrr}
\hline Model & WER & Params & FLOPs & Cost Reduct. \\
\hline Baseline & - & $42.7 \mathrm{M}$ & $11.1 \mathrm{~B}$ & - \\
Baseline Small & $+3.4 \%$ & $30.2 \mathrm{M}$ & $7.86 \mathrm{~B}$ & $29.1 \%$ \\
Bifocal & $\mathbf{- 1 . 9 \%}$ & $\mathbf{4 8 . 9 M}$ & $\mathbf{7 . 8 6 B}$ & $\mathbf{2 9 . 1 \%}$ \\
Bifocal (No Proj.) & $+3.4 \%$ & $46.3 \mathrm{M}$ & $7.85 \mathrm{~B}$ & $29.2 \%$ \\
Trifocal A & $+23.6 \%$ & $55.1 \mathrm{M}$ & $4.74 \mathrm{~B}$ & $57.3 \%$ \\
Trifocal B & $+27.0 \%$ & $55.1 \mathrm{M}$ & $3.70 \mathrm{~B}$ & $66.7 \%$ \\
Trifocal C & $+28.1 \%$ & $55.1 \mathrm{M}$ & $3.47 \mathrm{~B}$ & $68.7 \%$ \\
\hline
\end{tabular}

Table 2: Model WER and RNN-T encoder compute cost. WER is recorded in relative terms against a standard baseline model.

The key takeaway from our experiments is that even though the Bifocal encoder is $\sim 10 \%$ larger, it requires $\sim 30 \%$ fewer computations than the baseline. Meanwhile, the Bifocal model outperforms the baseline, small baseline, and No Projection Bifocal RNN-Ts, besting the small RNN-T's WER by 5\% while matching its FLOPs.

All three Trifocal models continued to improve upon compute cost but clearly pay a strikingly steep price in accuracy degradation. Despite this, we find it essential to include the Trifocal degradation results because they highlight two important considerations. First, they emphasize that the central design feature of the Bifocal RNN-T, utilizing keyword spotting, is justified as an effective way to switch between encoders. Arbitrary switching mid-stream, like the Trifocal models, is unlikely to retain accuracy. Second, the difference in WER between Trifocal A and that of B and C shows it is not the frequency of encoder switching but rather when and what fraction of frames are processed using the small encoder (A's 50\% post-WW compared to B and C's 66\%) that is the determining factor in predictive performance. This observation reinforces that success is dictated by where to strategically use the small encoder like we do with the Bifocal design.

\section{CONCLUSION}

We present an extension of the RNN-T architecture, Bifocal RNN$\mathrm{T}$, designed for improved inference-time latency for on-device ASR. By exploiting keyword spotting, we show that the Bifocal architecture improves encoder inference cost by $30 \%$ while matching baseline predictive performance. The technique is flexible and can be combined with other latency reduction techniques, such as sparsification and quantization. In future work we would like to see the approach extended to the decoder, experimented with different topologies, and tried with alternative and learnable switching schedules. 


\section{REFERENCES}

[1] Ian McGraw, Rohit Prabhavalkar, Raziel Alvarez, Montse Gonzalez Arenas, Kanishka Rao, David Rybach, Ouais Alsharif, Hasim Sak, Alexander Gruenstein, Francoise Beaufays, and Carolina Parada, "Personalized speech recognition on mobile devices," IEEE International Conference on Acoustics, Speech and Signal Processing (ICASSP), pp. 5955-5959, 2016.

[2] Jinyu Li, Hu Hu, and Yifan Gong, "Improving RNN transducer modeling for end-to-end speech recognition," IEEE International Conference on Acoustics, Speech and Signal Processing (ICASSP), pp. 114-121, 2019.

[3] Tara N. Sainath, Yanzhang He, Bo Li, Arun Narayanan, Ruoming Pang, et al., "A streaming on-device end-to-end model surpassing server-side conventional model quality and latency," IEEE International Conference on Acoustics, Speech and Signal Processing (ICASSP), pp. 6059-6063, 2020.

[4] Alex Graves, "Sequence transduction with recurrent neural networks," International Conference on Machine Learning (ICML), 2012.

[5] Alex Graves, Abdel-rahman Mohamed, and Geoffrey Hinton, "Speech recognition with deep recurrent neural networks," IEEE International Conference on Acoustics, Speech and Signal Processing (ICASSP), pp. 6645-6649, 2013.

[6] Alex Graves and Navdeep Jaitly, "Towards end-to-end speech recognition with recurrent neural networks," 31st International Conference on Machine Learning (ICML), pp. 37713779, 2014.

[7] William Chan, Navdeep Jaitly, Quoc V. Le, and Oriol Vinyals, "Listen, attend and spell," IEEE International Conference on Acoustics, Speech and Signal Processing (ICASSP), pp. 49604964, 2016.

[8] Hagen Soltau, Hank Liao, and Hasim Sak, "Neural speech recognizer: acoustic-to-word LSTM model for large vocabulary speech recognition," Proceedings of the Annual Conference of the International Speech Communication Association (INTERSPEECH), pp. 3707-3711, 2017.

[9] Raziel Alvarez, Rohit Prabhavalkar, and Anton Bakhtin, "On the efficient representation and execution of deep acoustic models," Proceedings of the Annual Conference of the International Speech Communication Association (INTERSPEECH), pp. 2746-2750, 2016.

[10] Yuriy Mishchenko and Chris Beauchene, "Training for smallfootprint keyword spotting," IEEE International Conference On Machine Learning And Applications (ICMLA), pp. 706$711,2018$.

[11] Hieu Duy Nguyen, Anastasios Alexandridis, and Athanasios Mouchtaris, "Quantization aware training with absolute-cosine regularization for automatic speech recognition," Proceedings of the Annual Conference of the International Speech Communication Association (INTERSPEECH), 2020.

[12] Yi Yang, Andy Chen, Xiaoming Chen, Jiang Ji, Zhenyang Chen, Yan Dai, and And Others, "Deploy large-scale deep neural networks in resource constrained IoT devices with local quantization region," arXiv preprint arXiv:1805.09473, 2018.

[13] Michael H. Zhu and Suyog Gupta, "To prune, or not to prune: exploring the efficacy of pruning for model compression," 6th
International Conference on Learning Representations, ICLR 2018 - Workshop Track Proceedings, 2018.

[14] Ruoming Pang, Tara N. Sainath, Rohit Prabhavalkar, Suyog Gupta, Yonghui Wu, Shuyuan Zhang, and Chung Cheng Chiu, "Compression of end-to-end models," Proceedings of the Annual Conference of the International Speech Communication Association (INTERSPEECH), , no. September, pp. 27-31, 2018.

[15] Yuan Shangguan, Jian Li, Qiao Liang, Raziel Alvarez, and Ian McGraw, "Optimizing speech recognition for the edge," arXiv preprint arXiv:1909.12408, 2019.

[16] Klaus Greff, Rupesh K. Srivastava, Jan Koutnik, Bas R. Steunebrink, and Jurgen Schmidhuber, "LSTM: a search space odyssey," IEEE Transactions on Neural Networks and Learning Systems, vol. 28, no. 10, pp. 2222-2232, 2017.

[17] Sepp Hochreiter and J Urgen Schmidhuber, "Long short term memory," Neural Computation, vol. 9, no. 8, pp. 1735-1780, 1997.

[18] Tao Lei, Yu Zhang, Sida I. Wang, Hui Dai, and Yoav Artzi, "Simple recurrent units for highly parallelizable recurrence," Proceedings of the 2018 Conference on Empirical Methods in Natural Language Processing, EMNLP 2018, pp. 4470-4481, 2018.

[19] Yanzhang He, Tara N. Sainath, et al., "Streaming end-toend speech recognition for mobile devices," IEEE International Conference on Acoustics, Speech and Signal Processing (ICASSP), 2019.

[20] Ming Sun, David Snyder, Yixin Gao, Varun Nagaraja, Mike Rodehorst, Sankaran Panchapagesan, Nikko Strom, Spyros Matsoukas, and Shiv Vitaladevuni, "Compressed time delay neural network for small-footprint keyword spotting," Proceedings of the Annual Conference of the International Speech Communication Association (INTERSPEECH), pp. 3607-3611, 2017.

[21] Sercan O. Arik, Markus Kliegl, Rewon Child, Joel Hestness, Andrew Gibiansky, Chris Fougner, Ryan Prenger, and Adam Coates, "Convolutional recurrent neural networks for smallfootprint keyword spotting," Proceedings of the Annual Conference of the International Speech Communication Association (INTERSPEECH), pp. 1606-1610, 2017.

[22] Alex Graves, Santiago Fernández, Faustino Gomez, and Jürgen Schmidhuber, "Connectionist temporal classification: labelling unsegmented sequence data with recurrent neural networks," 23rd international conference on Machine learning (ICML), pp. 369-376, 2006.

[23] Alex Graves, "Adaptive computation time for recurrent neural networks," arXiv preprint arXiv:1603.08983, pp. 1-19, 2016.

[24] Yacine Jernite, Edouard Grave, Armand Joulin, and Tomas Mikolov, "Variable computation in recurrent neural networks," 5th International Conference on Learning Representations, ICLR 2017 - Conference Track Proceedings, pp. 1-12, 2017.

[25] Taku Kudo and John Richardson, "SentencePiece: A simple and language independent subword tokenizer and detokenizer for neural text processing," Conference on Empirical Methods in Natural Language Processing: System Demonstrations, Proceedings (EMNLP), pp. 66-71, 2018. 